\title{
Quimioterapia neoadyuvante en un timoma invasor. Cirugía curativa con neumonectomía izquierda en bloque
}

\author{
A. F. Honguero Martínez, A. Arnau Obrer, M. Estors Guerrero, A. Juárez Marroquí1 ${ }^{1}$ J. Garde Noguera ${ }^{1}$, \\ J. A. Ros Lucas², D. Pérez Alonso ${ }^{3}$, A. Cantó Armengod
}

\section{Resumen}

Presentamos un paciente con un timoma invasor como hallazgo en un estudio radiológico por disnea progresiva. Tras el diagnóstico histológico fue tratado con quimioterapia (Qt) neoadyuvante consiguiendo una reducción del volumen tumoral del 25\%. El tratamiento quirúrgico consistió en una exéresis en bloque del timoma junto con el pulmón izquierdo por afectación de los vasos pulmonares. La combinación de la Qt neoadyuvante con la cirugía posibilitan un tratamiento con intención curativa.

\section{Palabras clave:}

Timoma. Pneumonectomía. Quimioterapia neoadyuvante.

Oncología, 2006; 29 (1):39-42

Servicio de Cirugía Torácica. Hospital General Universitario de Valencia.

${ }^{1}$ Servicio de Oncología Médica. Hospital General Universitario de Valencia.

${ }^{2}$ Servicio de Neumología. Hospital Universitario Virgen de la Arrixaca. Murcia.

${ }^{3}$ Servicio de Cirugía Torácica. Hospital Insular de las Palmas. 


\section{Summary}

We present a patient suffering from an invasive thymoma discovered in a chest roentgenogram because of progressive dyspnea. Neoadjuvant chemotherapy was used after histological diagnosis, observing a tumor volume decrease of $25 \%$. The surgical treatment consisted of the thymoma en bloc resection, and a left side pneumonectomy due to affected pulmonary vessels. The combination of neoadjuvant chemotherapy and surgery is applied as a curative intention therapy.

Key words: Thymoma. Pneumonectomy. Neoadjuvant chemotherapy.

\section{Introducción}

El timoma es un tumor poco frecuente. Se estiman menos de 2000 casos/año en los Estados Uni$\operatorname{dos}^{1}$. Su frecuencia es igual para ambos sexos y hay un pico de incidencia entre la cuarta y quinta décadas de la vida. En la mayoría de las ocasiones suele ser asintomático y es un hallazgo casual en una exploración radiológica. Pero otras veces hay clínica inespecífica como tos, dolor o disnea debido a la compresión que ocasiona sobre las estructuras vecinas. Suele tener un crecimiento lento y generalmente no metastatiza a distancia. En el 30-40\% de los pacientes con timoma se asocia la miastenia gravis (MG) pero sólo el 10-20\% de los pacientes con MG presentan un timoma ${ }^{2}$. El pronóstico viene determinado básicamente por el estadio y la cirugía completa. Presentamos un paciente con un timoma invasor en el que la quimioterapia (Qt) neoadyuvante y la cirugía completa permitieron un tratamiento curativo.

\section{Caso clínico}

Varón de 49 años con clínica de tos, escasa expectoración y aumento de su disnea que de ser a máximos esfuerzos se hizo a moderados esfuerzos con 3 semanas de evolución.

Antecedentes personales: exfumador desde hace 5 años (1.5 paq/día), hipertensión arterial, hipertensión ocular, operado de fractura de fémur y tibia hace 13 años complicada con osteomielitis, herniorrafia umbilical y apendicitis.

Exploración física: no fiebre, no dolor torácico, eupneico en reposo, no síndrome constitucional, disminución de la ventilación en el hemitórax izquierdo. Peso=73,4 Kg, altura=1,69 m.

Analítica: leucocitosis leve $\left(11.3 \times 10^{\wedge} 9 / 1\right)$ y ligera neutrofilia $(74.7 \%)$, bioquímica y coagulación: normales. Gasometría arterial: $\mathrm{pH}=7.41, \mathrm{pO} 2=74.0$ $\mathrm{mmHg}, \mathrm{pCO} 2=36.3 \mathrm{mmHg}$. Rx-TC: masa de contornos regulares de $10.8 \times 8.4 \mathrm{~cm}$ en el mediastino anterior izquierdo con extensión al hilio ipsilateral comprimiéndolo, derrame pleural izquierdo moderado. Fibrobroncoscopia: no tumoración endobronquial, hiperemia de la mucosa difusa. Pruebas de función respiratoria: FEV $1=2010$ 1.(59\%), $\mathrm{FVC}=2290$ 1.(54.8\%), índice de Tiffenau $=87.8 \%$. PAAF: células epiteliales con discreto grado de atipia y abundantes linfocitos, presencia de histiocitos, células gigantes multinucleadas y eosinófilos, algunas áreas de necrosis (citología sugerente de timoma).

El paciente fue sometido a biopsia por toracoscopia: nódulos en la pleura parietal y diafragmática, nódulos pulmonares en LII (informe de anatomía patológica intraoperatoria de tejido linfoide y material fibrohemorrágico), evacuación de $2000 \mathrm{ml}$ de derrame pleural y observación de una masa tumoral en mediastino anterior.

Los resultados anatomopatológicos definitivos fueron:

- Citología del líquido pleural: hiperplasia mesotelial reactiva

- Biopsia de pleura parietal y diafragmática: tejido fibroso hialinizado, tejido fibroso y muscular

- Biopsia de la masa mediastínica: células epiteliales positivas para citoqueratina y CAM 5.2, células linfoides positivas para CD-3 compatibles con el diagnóstico de timoma cortical. 
TABLA I

\begin{tabular}{|c|c|}
\hline Estadio de Masaoka & Grado de la enfermedad \\
\hline I & $\begin{array}{l}\text { Macroscópicamente: completa- } \\
\text { mente encapsulado } \\
\text { Microscópicamente: sin infiltra- } \\
\text { ción capsular }\end{array}$ \\
\hline II & $\begin{array}{l}\text { Invasión macroscópica del tejido } \\
\text { adiposo vecino o pleura mediastí- } \\
\text { nica. } \\
\text { Microscópicamente: invasión cáp- } \\
\text { sular y/o invasión de la grasa cir- } \\
\text { cundante o de la pleura }\end{array}$ \\
\hline III & $\begin{array}{l}\text { Invasión de órganos (pericardio, } \\
\text { pulmones, grandes vasos, corazón) }\end{array}$ \\
\hline IVa & Implantes pleurales o pericárdicos \\
\hline $\mathrm{IVb}$ & $\begin{array}{l}\text { Metástasis hematógenas y/o linfá- } \\
\text { ticas }\end{array}$ \\
\hline
\end{tabular}

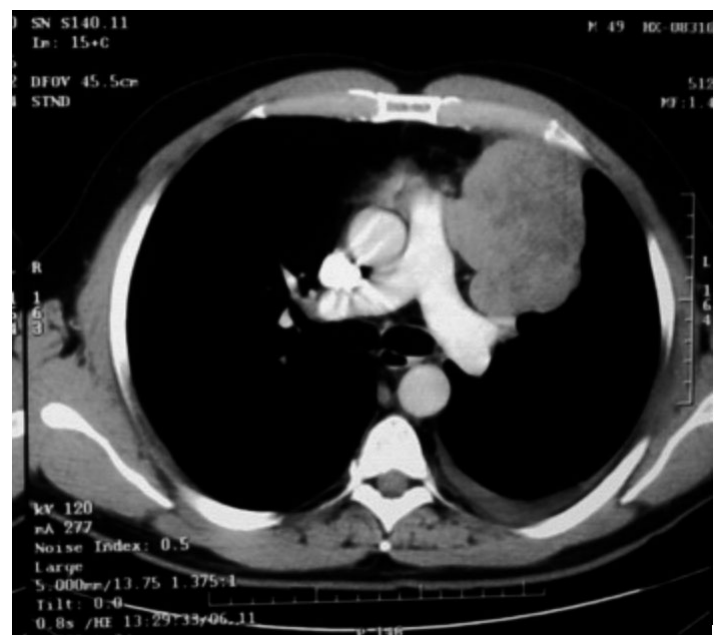

Figura 1. Tumor antes de la Qt. Derrame pleural asociado.

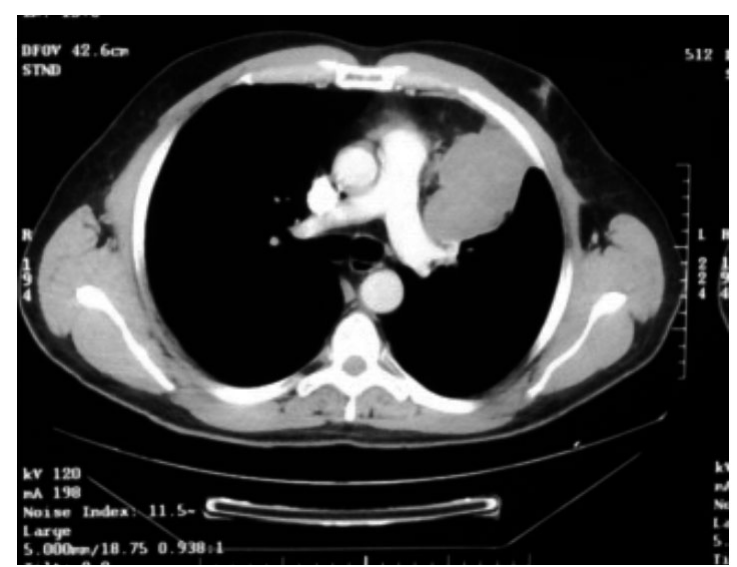

Figura 2. Tumor después de la Qt (reducción de su tamaño).
Por tratarse de un timoma invasor (estadío-III de Massaoka), se planeó una tratamiento con Qt neoadyuvante, cirugía y posterior radioterapia.

La Qt consistió en: adriamicina $50 \mathrm{mg} / \mathrm{m}^{2}$ y CDDP $100 \mathrm{mg} / \mathrm{m}^{2}$ en tres ciclos con intervalo de 21 días. En el segundo y tercer ciclos se disminuyó la dosis de CDDP a $75 \mathrm{mg} / \mathrm{m}^{2}$ por vómitos y somnolencia.

En la TC de control tras la Qt se observó una reducción del tamaño aproximadamente en un $25 \%$ a expensas de la porción periférica manteniendo la prolongación hacia el hilio pulmonar: $7.9 \times 6.3 \mathrm{~cm}$ infiltrando la pleura mediastínica y contactando con la arteria pulmonar izquierda.

En la cirugía se realizó la extirpación en bloque del timoma junto a una neumonectomía izquierda y linfadenectomía mediastínica englobando los ganglios de las áreas 5, 6, 7, 8 y 9. Los nervios frénico y vago izquierdos fueron seccionados por invasión tumoral.

En el postoperatorio inmediato el paciente presentó una anemia bien tolerada sin precisar transfusión y fue dado de alta al quinto día de la intervención.

Los resultados de la anatomía patológica fueron: todas las adenopatías libres de tumor, se confirma el diagnóstico previo de timoma y un índice de proliferación tumoral del $20 \%$.

\section{Discusión}

El tratamiento del timoma se basa principalmente en la cirugía ya que uno de los principales factores que influyen en la supervivencia a largo plazo es la resección completa ${ }^{5}$. Para la estadificación del timoma no hay ningún sistema estándar pero se suele utilizar la clasificación de Masaoka ${ }^{3}$ (Tabla I) Actualmente, ante un timoma invasor, el arsenal terapéutico es multidisciplinario: Qt, radioterapia (Rt) y cirugía. Hoy día no hay ningún consenso establecido sobre cuál es la mejor combinación terapéutica y existe disparidad de criterios sobre la adyuvancia.

La Qt neoadyuvante es útil, consigue una citorreducción y facilita la resección completa. Se basa en cis-platino asociado a otros quimioterápicos como adriamicina, doxorrubicina, ciclofosfamida, vincristina, etc. Con la poliquimioterapia se obtiene mejor respuesta tumoral ${ }^{4}$ que en nuestro caso fue una re- 
ducción del 25\%. Estos fármacos se administran distribuidos en varios ciclos antes de la cirugía que tiene lugar unas pocas semanas posteriormente (cuatro en nuestro caso). La administración de Qt intrapleural se ha descrito en caso de implantes pleurales o de la rotura del tumor durante la cirugía 5 . La recidiva del timoma invasor suele afectar más a la pleura que al mediastino por lo que estos pacientes se podrían beneficiar de la Qt adyuvante ${ }^{5}$.

En la cirugía, durante la manipulación del tumor hay que evitar su laceración para reducir la posibilidad de diseminación. De este modo, la resección se realizó en bloque junto con la neumonectomía por infiltración de los vasos principales del pulmón izquierdo. El nervio frénico si está afecto se debe seccionar a menos que el paciente presente una limitación respiratoria o se trate de una afectación bilateral de modo que se respetaría dejando una pastilla tumoral lo más pequeña posible (debulking). En caso de seccionar el frénico es conveniente la plicatura diafragmática para evitar un ascenso importante del diafragma que arrastraría consigo a las vísceras abdominales comprimiendo así el mediastino y sus órganos vitales.

El tratamiento con Rt es un tema más controvertido en el timoma invasor. Se ha venido utilizando como adyuvancia de forma rutinaria tras la cirugía con intención curativa (aislada o junto con Qt adyuvante). La Rt puede producir consecuencias graves como neumonitis actínica, fibrosis pulmonar, cáncer de esófago, cardiomiopatía restrictiva, arritmias, etc. Estos efectos junto con los resultados obtenidos por algunos autores han hecho que su utilidad sea cuestionada $^{5}$. La Rt desempeña un papel importante en pacientes en los que no ha sido posible una resección completa o con reducidos márgenes de seguridad. Nosotros la utilizamos como adyuvancia siguiendo nuestro protocolo. Otros grupos también defienden la Rt adyuvante por obtener mejores resultados tras la Qt neoadyuvante y cirugía quizás por un efecto de radiosensibilidad inducida con la $\mathrm{Qt}^{6}$. En cualquier caso, dado que el timoma es una patología rara por su reducida frecuencia es difícil establecer unas pautas ideales para su tratamiento más eficiente aunque el pilar fundamental sigue siendo hoy día la cirugía.

\section{Bibliografía}

1. SEER: Surveillance, Epidemiology, and End Results (SEER) cancer incidence public-use database, 1973-1996. Baltimore, MD, National Cancer Institute, DCCPS, Surveillance Research Programm, Cancer Statistics Branch, released April 1999.

2. Mark I. Block: Thymic tumors. In F. Griffith Pearson, Joel D. Cooper, Jean Deslauriers et al. (eds). Thoracic Surgery, 2nd ed. Churchill Livingstone; Philadelphia (USA) 2002, p. 1682-1710.

3. Masaoka A, Monden Y, Nakahara K, et al. Follow-up study of thymomas with special reference to the clinical stages. Cancer 1981; 48: 2485-99.

4. Loehrer PJ, Kim K, Aisner SC, et al. Cisplatin, doxorubicin, and cycolophosphamide plus thoracic radiation therapy for limited-stage unresectable thymoma: an intergroup trial. J Clin Oncol 1997; 15: 3093-3099.

5. Mangi AA, Wain JC, Donahue DM, Grillo HC, Mathisen DJ, Wright CD. Adjuvant radiation of stage III thymoma: is it necessary? Ann Thorac Surg 2005; 79: 1834-1839.

6. Lucchi M, Ambrogi MC, Duranti L, Basolo F, Fontanini G, Angeletti CA, Mussi A. Advanced stage thymomas an thymic carcinomas: results of multimodality treatments. Ann Thorac Surg 2005; 79: 1840-1844.

Correspondencia:

Dr. A. F. Honguero

Servicio Cirugía Torácica

Hospital General Universitario de Valencia

Avda. Tres Cruces, 2

E-46014 Valencia

tonyhonguero@yahoo.es 